\title{
A new rheological model and its application in Mountain Tunnelling
}

\author{
Zhenchang Guan ${ }^{1, *}$, Yujing Jiang ${ }^{2}$, Yoshihiko Tanabashi ${ }^{2}$ and Hongwei Huang ${ }^{3}$ \\ ${ }^{1}$ Graduate School of Science and Technology, Nagasaki University, Nagasaki, Japan \\ ${ }^{2}$ Department of Civil Engineering, Faculty of Engineering, Nagasaki University, Nagasaki, Japan \\ ${ }^{3}$ Department of Geotechnical Engineering, Tongji University, Shanghai, China
}

\begin{abstract}
The time-dependent features of soft rock, named rheology generally, should be taken into account in the long-term design and maintenance of mountain tunnels. Based on the classic Burger-MC rheological model, a Burger-Deterioration rheological model is proposed in this paper and is implemented in the numerical codes FLAC ${ }^{3 \mathrm{D}}$. A deterioration threshold and two deterioration ratios are introduced in this model to consider the time-dependent strength deterioration aspect of the rock mass. The proposed model is applied to an engineering instance (Ureshino Tunnel Line I, Nagasaki, Japan) to account for the delayed deformations that occurred after its completion since Nov. 1992. The delayed crown settlement and invert upheaval computed from simulations are featured by an exponential characteristic and a stair-typed characteristic, respectively, which agree well with the in-site monitoring data qualitatively. In addition, the realistic rheological parameters involved in the proposed model can be back-analyzed from the in-site monitoring data.
\end{abstract}

Key words: Rheology, Burger-Deterioration model, Delayed deformation, NATM

\section{Introduction}

The New Austrian Tunneling Method (NATM) has innovated the concept of conventional tunnelling from resisting the passive earth pressure to helping the ground support itself, and has been widely used in mountain tunnel excavation (Carranza-Torres and Fairhurst, 1999; Oreste, 2003; Guan et al., 2006). However, this method should be considered circumspectly when applied to the soft rock, which exhibits strain softening behaviors and time-dependent features. The tunnels excavated in soft rock would occasionally experience a large delayed deformation that might lead to a delayed failure of structure. Therefore, the time-dependent features of soft rock, named rheology generally, should be taken into account in the long-term design and maintenance of mountain tunnels.

The rheological characteristics of some types of rock have been comprehensively studied through a great deal of specified creep, relaxation and quasistatic compression tests (Maranini and Brignoli, 1999; Li and Xia, 2000; Fabre and Pellet, 2006). According to the results from laboratory (or in-site) tests and the experience from engineering practice, many rheological models have been proposed to

\footnotetext{
${ }^{*}$ Corresponding author: Graduate School of Science and Technology, Nagasaki University. 1-14 Bunkyo Machi, Nagasaki 852-8521 Japan.

Email: gaussto@hotmail.com
} 
account for the time-dependent features of rock mass from manifolds. These models can be generally divided into two categories: the classic viscoplastic models and the viscoplastic-damaged models. The constitutive laws in the classic viscoplastic models try to relate the current strain rate to the current stress (and/or stress rate) directly. Particularly, the relationship between the deviatoric strain rate and the deviatoric stress (and/or stress rate) can be schematically represented by a series of spring, dashpot and plastic slider that connected in parallel and/or in series. This category of rheological model is represented by the Burger-MC model, the Bingham model, the power law MC model etc. (Hudson and Harrison, 1997; Itasca Consulting Group, 1997).

The constitutive laws in the viscoplastic-damaged models are based on the principle of strain and energy equivalence and are derived from a standard thermodynamic dissipation potential. Many researchers have contributed theirs pioneering works on this issue (Pellet et al., 2005; Bhandari and Inoue, 2005; Shao et al., 2006). Generally, the dissipation potential consists of two independent potentials, which correspond to the strain softening (i.e. plasticity) process and the damaging process, respectively. When these two potentials are well defined, by applying the normality rule, the plastic strain rate and the damage evolution rate can be formulated as the potential's first-order difference with respect to the current stress and the thermodynamic force, respectively.

In fact, the viscoplastic-damaged models are first proposed to account for the tertiary creep phase phenomenon that occurred in some rock specimens creep tests, where the classic viscoplastic models failed to do. However, the viscoplastic-damaged models are generally more complicated than the former ones. Based on the classic Burger-MC model, a Burger-Deterioration model is proposed in this paper. Instead of the complicated damage mechanics, a deterioration threshold and two deterioration ratios are introduced in this model to consider the time-dependent strength deterioration aspect of rock equivalently. The proposed model is implemented in the numerical codes FLAC ${ }^{3 \mathrm{D}}$, and is applied to an engineering instance (Ureshino Tunnel Line I) to account for the delayed deformations that occurred after its completion since Nov. 1992.

\section{The Burger-Deterioration Model}

\subsection{The classic Burger-MC model}

The constitutive laws of the classic Burger-MC model are characterized by an elastoplastic volumetric behavior and a viscoplastic deviatoric behavior. The deviatoric behavior can be schematically illustrated in Fig.1, where a Kelvin unit characterized by its shear modulus $G^{K}$ and viscosity $\eta^{K}$, a Maxwell unit characterized by its shear modulus $G^{M}$ and viscosity $\eta^{M}$ and a Mohr-Coulomb plastic unit characterized by its cohesion $c$, friction angle $\phi$ and dilation angle $\psi$ are connected in series and subjected to a certain deviatoric loading jointly. Consequently, the deviatoric strain rate partitioning is formulated as Eq. (1). And the constitutive laws of the deviatoric behavior 
for these three units are formulated as Eqs. (2)-(4), respectively, while the constitutive laws of the volumetric behavior are formulated as Eq. (5).

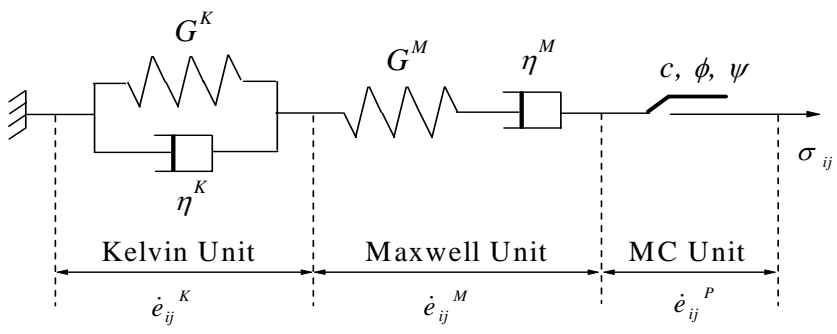

Fig. 1. Schematical representation of the deviatoric behavior of Burger-MC rheological model

$$
\begin{aligned}
& \dot{e}_{i j}=\dot{e}_{i j}^{K}+\dot{e}_{i j}^{M}+\dot{e}_{i j}^{P} \\
& s_{i j}=2 \eta^{K} \dot{e}_{i j}^{K}+2 G^{K} e_{i j}^{K} \\
& \dot{e}_{i j}^{M}=\frac{\dot{s}_{i j}}{2 G^{M}}+\frac{s_{i j}}{2 \eta^{M}} \\
& \dot{e}_{i j}^{P}=\lambda \frac{\partial g}{\partial \sigma_{i j}}-\frac{\dot{\varepsilon}_{k k}^{P}}{3} \delta_{i j} \text { with } \dot{\varepsilon}_{k k}^{P}=\lambda\left[\frac{\partial g}{\partial \sigma_{11}}+\frac{\partial g}{\partial \sigma_{22}}+\frac{\partial g}{\partial \sigma_{33}}\right] \\
& \dot{\sigma}_{k k}=3 K\left(\dot{\varepsilon}_{k k}-\dot{\varepsilon}_{k k}^{P}\right)
\end{aligned}
$$

where $e_{i j}$ and $s_{i j}$ are the deviatoric components derived from the strain tensor and the stress tensor, respectively; $\varepsilon_{k k}$ and $\sigma_{k k}$ are the volumetric components of the strain and stress tensors. The superscripts $K, M$ and $P$ denote the Kelvin, Maxwell and MC plastic components of the corresponding variables. $g$ is the plastic potential for MC unit, and $\lambda$ is a multiplier that can be eliminated in the calculation afterwards. $K$ is the bulk modulus of the rock mass.

On the other hand, the stress state should be enveloped by a failure criterion. For MC unit, the failure criterion $f$ and the plastic potential $g$ generally can be expressed as

$$
\begin{aligned}
& f=\sigma_{1}-\sigma_{3} \frac{1+\sin \phi}{1-\sin \phi}+2 c \sqrt{\frac{1+\sin \phi}{1-\sin \phi}} \\
& g=\sigma_{1}-\sigma_{3} \frac{1+\sin \psi}{1-\sin \psi}
\end{aligned}
$$

where $\sigma_{1}$ and $\sigma_{3}$ are the major and the minor principal stresses; $c, \phi$ and $\psi$ are the cohesion, the friction angle and the dilation angle of MC unit. 


\subsection{The Burger-Deterioration model}

Some types of rock specimens would experience three different phases in some creep compression tests, if the constant stress applied on the specimens is larger than a threshold (the so-called long-term strength). The strain rate will attenuate in primary phase, stabilize in secondary phase and accelerate in tertiary phase, which lead to a delayed failure finally. The viscoplastic-damaged models incorporate the damage mechanics with the viscoplastic mechanics to account for the tertiary creep phase phenomenon. However, there is not a well-recognized one among the researchers, since the damage mechanics would differ from each other due to different types of rock mass (such as brittle rock and argillaceous rock).

Instead of introducing the complicated damage mechanics, a Burger-Deterioration rheological model is proposed in this paper. Its framework is the same with the Burger-MC model and it just simply assumes that the cohesion $c$ and the friction angle $\phi$ will decrease with time, regardless of whether the loss of strength is caused by cycle loading fatigue, by clay mineral hydration or by some other reasons. It is assumed that the loss of strength is controlled by its current stress state; furthermore, there exists a threshold to initiate this kind of strength deterioration and a lower limit to circumscribe the strength deterioration

$$
\begin{aligned}
& \frac{d c}{d t}=-\omega_{c} R \quad\left(R \geq R_{t h r}, \quad c \geq c_{r e s}\right) \\
& \frac{d \phi}{d t}=-\omega_{\phi} R \quad\left(R \geq R_{t h r}, \quad \phi \geq \phi_{r e s}\right) \\
& R=\frac{\sigma_{1}-\sigma_{3}}{2 c \cos \phi+\left(\sigma_{1}+\sigma_{3}\right) \sin \phi}
\end{aligned}
$$

In the above equations, the parameter $R$ is named stress coefficient in this paper and indicates the "distance” from the current stress state to the MC failure envelope. When the stress coefficient is greater than a certain threshold $R_{t h r}$, the rock strength initiates to deteriorate. The multipliers $\omega_{c}$ and $\omega_{\phi}$ are two deterioration ratios that scale the increments of $c$ and $\phi$ by some certain proportions. $c_{\text {res }}$ and $\phi_{\text {res }}$ are residual cohesion and residual friction angle that can be estimated from conventional triaxial tests.

The Eqs. (1)-(8) that describe the constitutive laws of the Burger-Deterioration model can be implemented in the numerical codes FLAC ${ }^{3 \mathrm{D}}$ (Itasca Consulting Group, 1997). The FLAC ${ }^{3 \mathrm{D}}$ codes use an explicit time-marching scheme to find the solution of a problem. The equations of motions are solved to derive new velocities and displacements from forces and stresses. Velocities are then used to calculate strain rates, from which new stresses can be found through the constitutive equations. Although the proposed model is not included directly in the FLAC ${ }^{3 \mathrm{D}}$, s constitutive laws library, fortunately, the programmable language FISH can help to implement it indirectly under the framework of the classic Burger-MC model in such a way: reevaluating the new cohesion and 
friction angle for every element according to the element's current stress, before each new calculating cycle.

\section{Delayed Deformation Analysis and Discussions}

\subsection{The outline of the Ureshino Tunnel}

The construction of Ureshino Tunnel Line I in Nagasaki Expressway began from May 1990 and finished in Nov. 1992. However, the tunnel experienced a continuous converging during its service time. Therefore, reinforcement on the tunnel invert and its surrounding ground began in 2000 when the Ureshino Tunnel Line II was put into use. This continuous converging was considered to be relative with the rheological features of the surrounding rock mass. Therefore, the Burger-Deterioration model is applied to this engineering instance to account for the mechanics of delayed deformation that occurred after the tunnel's completion. A period of five years, from Nov. 1992 when Line I was completed to Nov. 1997 when Line II commenced working, is mainly focused in this paper.

The geological profile of Ureshino Tunnel Line I is schematically illustrated in Fig. 2. The cross section dimensions and the tunnel convergence monitoring positions, mainly including the displacements at the crown $\left(u_{c}\right)$, at the springline $\left(u_{s}\right)$ and at the invert $\left(u_{i}\right)$, are schematically illustrated in Fig. 3. In the longitudinal direction, as illustrated in Fig. 4, the span of each excavation cycle was $1.0 \mathrm{~m}$. The first lining and the rock bolt were installed immediately after each excavation cycle, and the second lining was cast in place after 25 excavation cycles were completed.

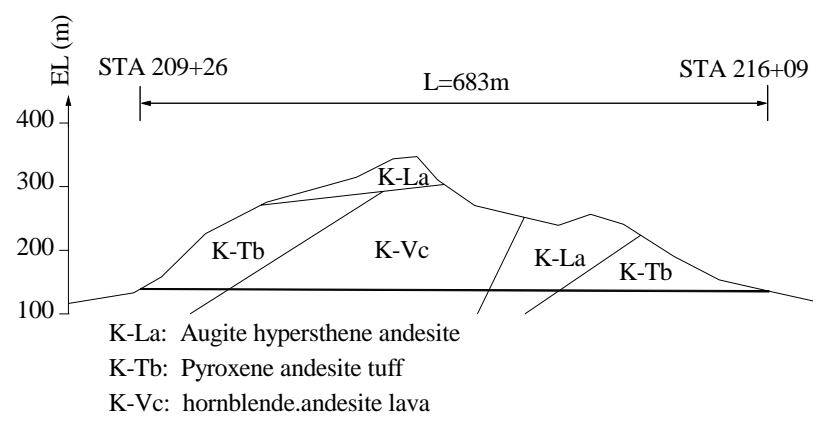

Fig. 2. Schematical representation of geological profile of Ureshino Tunnel

In this paper, a total of 30 excavation cycles (from STA 211+70 to STA 212+00) are simulated step by step in FLAC ${ }^{3 \mathrm{D}}$. The properties of the rock mass and the linings, as listed in Table 1 and Table 2 respectively, are referenced to the construction and monitoring reports on Ureshino Tunnel (Japan Highway Society, 2000). Notice that the rheological calculation switch is set off and the rheological parameters are unnecessary at this excavation stage, since the elastoplasticity is the overwhelming mechanics rather than the rheology. The simulations after excavation stage provide an "initial state" for the further rheological calculation. 
Table 1. The properties of the rock mass employed in numerical simulations.

\begin{tabular}{cc|cc}
\hline Properties & Value & Properties & Value \\
\hline$\rho\left(\mathrm{kg} / \mathrm{m}^{3}\right)$ & 2500 & $G^{K}(\mathrm{MPa})$ & N/A \\
$K(\mathrm{MPa})$ & 833 & $\eta^{K}(\mathrm{~Pa} \cdot \mathrm{s})$ & N/A \\
$G^{M}(\mathrm{MPa})$ & 385 & $\eta^{M}(\mathrm{~Pa} \cdot \mathrm{s})$ & N/A \\
$c(\mathrm{MPa})$ & 0.577 & $\omega_{c}(\mathrm{MPa} / \mathrm{y})$ & N/A \\
$\phi\left(\left(^{\circ}\right)\right.$ & 30.0 & $\omega_{\phi}\left({ }^{\circ} / \mathrm{y}\right)$ & N/A \\
$c_{\text {res }}(\mathrm{MPa})$ & 0.310 & $R_{\text {thr }}$ & N/A \\
$\phi_{\text {res }}\left({ }^{\circ}\right)$ & 25.5 & In-situ $\sigma_{z z}(\mathrm{MPa})$ & 8.0 \\
$\psi\left(\left(^{\circ}\right)\right.$ & 5.1 & In-situ $\sigma_{x x}(\mathrm{MPa})$ & 8.0 \\
\hline
\end{tabular}

Table 2. The properties of the linings employed in numerical simulations.

\begin{tabular}{ccc}
\hline & First Lining & Second Lining \\
\hline$E(\mathrm{MPa})$ & $1.0 \mathrm{e} 4$ & $2.0 \mathrm{e} 4$ \\
$\mu$ & 0.25 & 0.25 \\
$\sigma_{c}(\mathrm{MPa})$ & 15 & 30 \\
$\phi\left({ }^{\circ}\right)$ & 35 & 40 \\
$\psi\left({ }^{\circ}\right)$ & 3.0 & 3.0 \\
$t_{c}(\mathrm{~m})$ & 0.15 & 0.35 \\
\hline rock bolt & & first lining \\
\hline Lb=3000 & & tc=150 \\
\hline
\end{tabular}

Fig. 3. The cross section dimensions and the convergence monitoring positions

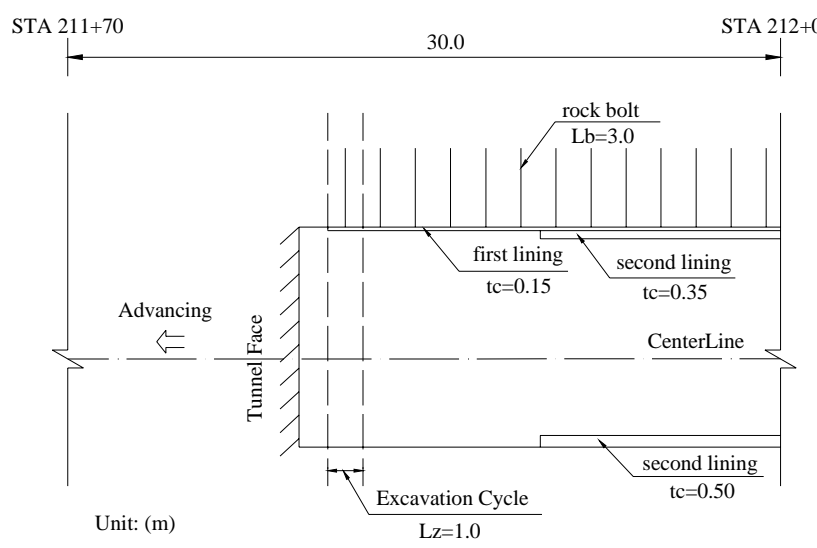

Fig. 4. Schematical representation of excavation cycling in construction process and numerical simulations 


\subsection{Delayed deformation characteristics}

Setting the rheological calculation switch on and using the Burger-Deterioration rheological model, the numerical simulations aforementioned proceed to rheological analyses focusing on the period from Nov. 1992 to Nov. 1997. Firstly, the deformation characteristics of each unit that consists the proposed model should be discussed respectively.

Shielding off the Maxwell unit and setting the deterioration ratios to zeros, the proposed model degenerates into a Kelvin-MC model, whose deformation characteristics are depicted in Fig. 5. Basically, both the crown settlement and the invert upheaval increase exponentially. The magnitude and the period of ultimate deformation are mainly controlled by $G^{K}$ and $\eta^{K}$, respectively. Similarly, shielding off the Kelvin unit and setting the deterioration ratios zeros, the proposed model degenerates into Maxwell-MC model, whose deformation characteristics are depicted in Fig. 6. Basically, both the crown settlement and the invert upheaval increase linearly, with their slopes controlled by $\eta^{M}$. In addition, it can be concluded from Figs. 5-6 that the viscoplastic mechanics of rock mass would cause a larger delayed convergence at the crown rather than at the invert.

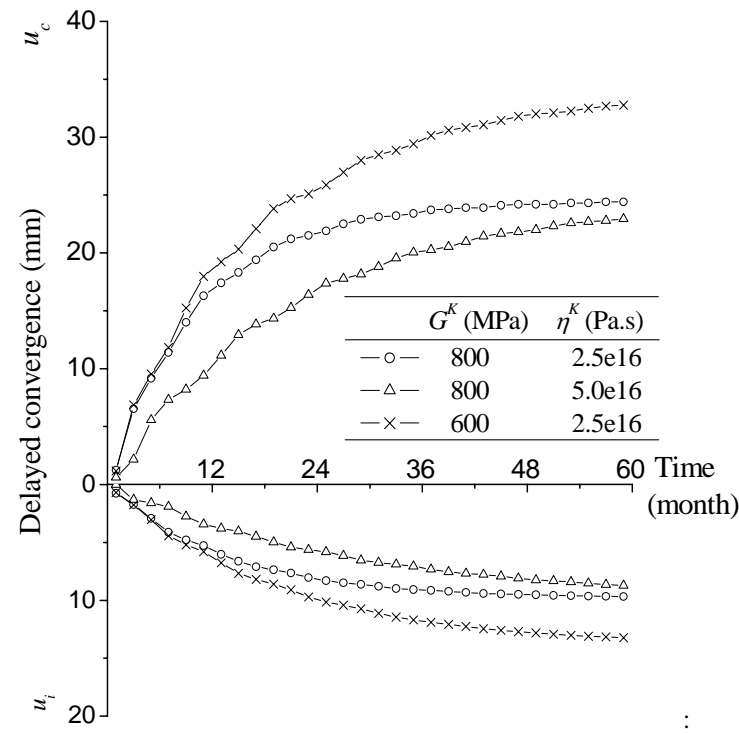

Fig. 5. The deformation characteristics of the Kelvin-MC model

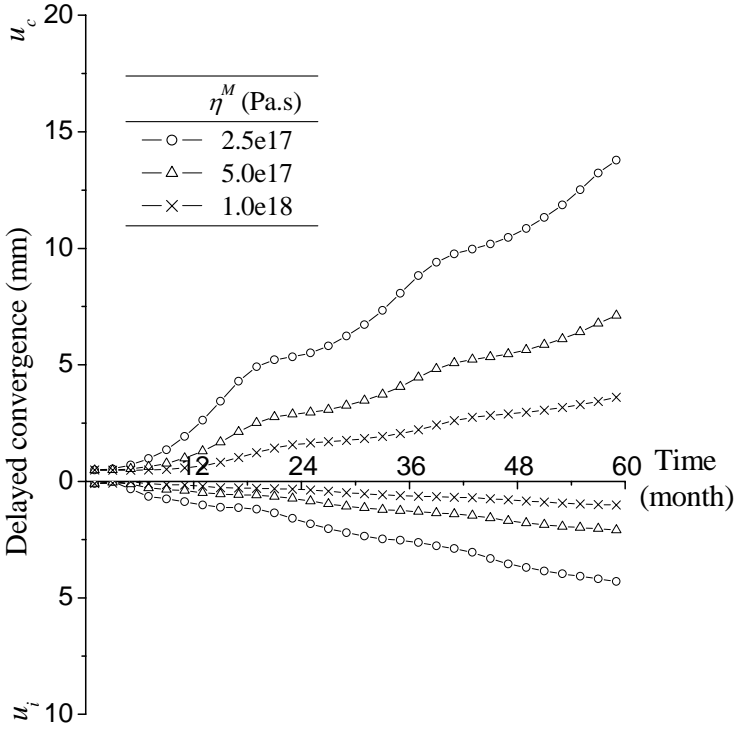

Fig. 6. The deformation characteristics of the Maxwell -MC model

When shielding off the Kelvin unit and Maxwell unit, the proposed model degenerates into a MC deterioration model, whose deformation characteristics are depicted in Fig. 7. On the occasion of low deterioration ratios, both the crown settlement and the invert upheaval increase linearly with small slopes. However, on the occasion of high deterioration ratios, the delayed deformations will accelerate and fail to converge, which would lead to a delayed failure of structure. In addition, it can be concluded that the strength deterioration of rock mass would cause a more significant delayed convergence at the invert rather than at the crown. 
In fact, the deformation characteristics of the Kelvin unit, the Maxwell unit and the MC deterioration unit correspond to the first, the second and the third phases in some creep tests, where the strain rate attenuates, stabilizes and accelerates respectively. It can be inferred that the deformation characteristics of the Burger-Deterioration model would compose of all these three components. Another three cases with different rheological parameters are studied, and the delayed deformations are partly depicted in Fig. 8. The characteristic of the crown settlement is basically the same with Fig. 5, since it is dominated by the viscoplastic mechanics. On the other hand, two comparatively mechanics, the viscoplasticity and the strength deterioration, influence the invert upheaval jointly, which leads to a stair-typed deformation characteristic as illustrated in Fig. 8. It is the stair-typed deformation characteristic that agrees with the monitoring data (the squared-line in Figs. 9-11) qualitatively, which implies the suitability of the proposed model to account for the delayed deformation occurred in Ureshino Tunnel Line I after its completion.

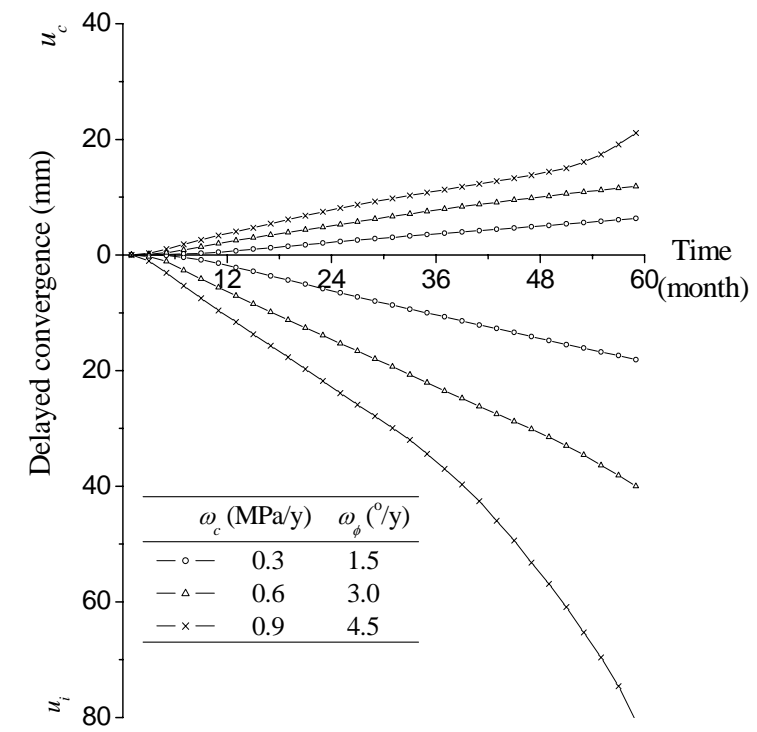

Fig. 7. The deformation characteristics of the

MC deterioration model $\left(R_{\text {thr }}=0.65\right)$

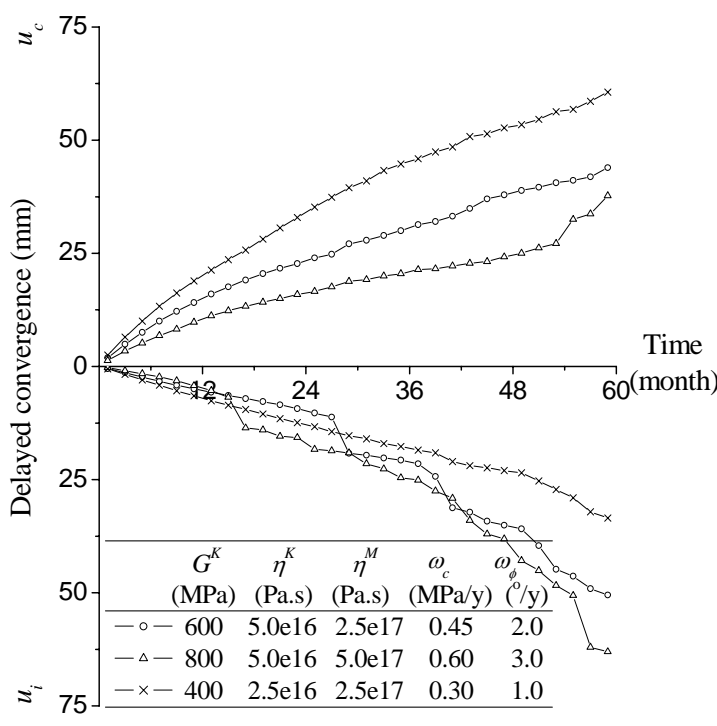

Fig. 8. The deformation characteristics of the Burger- Deterioration model. $\left(R_{\text {thr }}=0.65\right)$

\subsection{Parameter study}

Although the proposed model is able to account the delayed deformation mechanics in Ureshino Tunnel Line I qualitatively, another problem arises that how to evaluate the corresponding parameters involved in this model more realistic, since these rheological parameters usually are not ready for a certain engineering instance. It is suggested that they should be back-analyzed from the in-site monitoring data, if these data are available. Generally, the optimal method using artificial network is a powerful means for parameter identification (Pichler et al., 2003; Haykin, 2002). However, this issue is a problem far beyond the scope of this paper, thus in this paper, a simple 
means of weighted average is employed to evaluate the "realistic" parameters for the Burger-Deterioration model, according to the in-site monitoring data.

The influence of strength deterioration parameters $\omega_{\phi}, \omega_{c}$ and $R_{t h r}$ is highlighted in case group A, where the viscoplastic parameters are assumed constant and the strength deterioration parameters are taken some random values (see Appendix A for parameter evaluation). For each case, comparing the simulation results with the in-site monitoring data, the errors for the crown settlement and the invert upheaval in the $j^{\text {th }}$ case are defined as:

$$
\begin{aligned}
& e_{u c}{ }^{j}=\frac{\left\|\vec{u}_{c}^{j}-\vec{u}_{c}^{m o}\right\|}{\left\|\vec{u}_{c}^{m o}\right\|} \quad(1 \leq j \leq 10) \\
& e_{u i}{ }^{j}=\frac{\left\|\vec{u}_{i}^{j}-\vec{u}_{i}^{m o}\right\|}{\left\|\vec{u}_{i}^{m o}\right\|} \quad(1 \leq j \leq 10)
\end{aligned}
$$

In the above equations, $\vec{u}_{c}{ }^{j}\left(\vec{u}_{i}{ }^{j}\right)$ are $60 \times 1$ vectors recording the crown settlements (the invert upheavals) that computed from the $j^{\text {th }}$ case group A. $\vec{u}_{c}^{m o}\left(\vec{u}_{i}^{m o}\right)$ are their counterparts that measured from monitoring data during sixty months from Nov. 1992. $\|$ || is the denotation of norm calculation for vectors. Then, the weight for each case $w_{j}$, can be calculated according to these errors:

$$
w^{j}=\frac{\frac{2}{e_{u c}^{j}+e_{u i}^{j}}}{\sum_{j=1}^{10} \frac{2}{e_{u c}^{j}+e_{u i}^{j}}} \quad(1 \leq j \leq 10)
$$

Then, the weighted average of the rheological parameters can be obtained via Eq. (11).

$$
\vec{a}^{00}=\sum_{j=1}^{10} w^{j} \vec{a}^{j} \quad(1 \leq j \leq 10)
$$

In the above equation, $\vec{a}^{j}$ and $\vec{a}^{00}$ are $6 \times 1$ vectors recording the rheological parameters for the $j^{\text {th }}$ case group A and the weighted average case A00. Then employing $\vec{a}^{00}$ again into the same simulations, the results of the weighted average case A00 are delineated in Fig. 8. The case information of case group A, including properties, errors and weights, are summarized in Table 3. It can be concluded that the strength deterioration parameters influence the invert upheaval significantly rather than the crown settlement. 
Table 3. Case information of group A.

\begin{tabular}{c|cccccc}
\hline $\begin{array}{c}\text { Case } \\
\text { No. }\end{array}$ & $\begin{array}{c}\omega_{c} \\
(\mathrm{MPa} / \mathrm{y})\end{array}$ & $\begin{array}{c}\omega_{\phi} \\
(\mathrm{o} / \mathrm{y})\end{array}$ & $R_{t h r}$ & $\begin{array}{c}e_{u c} \\
(\%)\end{array}$ & $\begin{array}{c}e_{u i} \\
(\%)\end{array}$ & $\begin{array}{c}\text { Weight } \\
(\%)\end{array}$ \\
\hline A01 & 0.103 & 5.46 & 0.45 & 6.8 & 213.5 & 0.6 \\
A02 & 0.081 & 4.25 & 0.55 & 1.5 & 55.7 & 2.5 \\
A03 & 0.067 & 3.06 & 0.60 & 2.7 & 2.4 & 28.1 \\
A04 & 0.045 & 2.12 & 0.65 & 3.1 & 7.5 & 13.5 \\
A05 & 0.021 & 1.05 & 0.80 & 3.2 & 17.7 & 6.9 \\
A06 & 0.016 & 5.52 & 0.55 & 4.5 & 45.2 & 2.9 \\
A07 & 0.046 & 3.88 & 0.60 & 3.2 & 6.2 & 15.3 \\
A08 & 0.039 & 3.21 & 0.75 & 3.3 & 5.8 & 15.9 \\
A09 & 0.059 & 0.95 & 0.85 & 4.8 & 16.5 & 6.7 \\
A10 & 0.096 & 1.37 & 0.70 & 5.8 & 13.2 & 7.6 \\
\hline A00 & 0.054 & 2.79 & 0.66 & 3.8 & 3.6 & \\
\hline Group A: $G^{K}=800 \mathrm{MPa}, \eta^{K}=4.0 \mathrm{e} 16 \mathrm{~Pa} \cdot \mathrm{s}, \eta^{M}=5.0 \mathrm{e} 17 \mathrm{~Pa} \cdot \mathrm{s}$
\end{tabular}

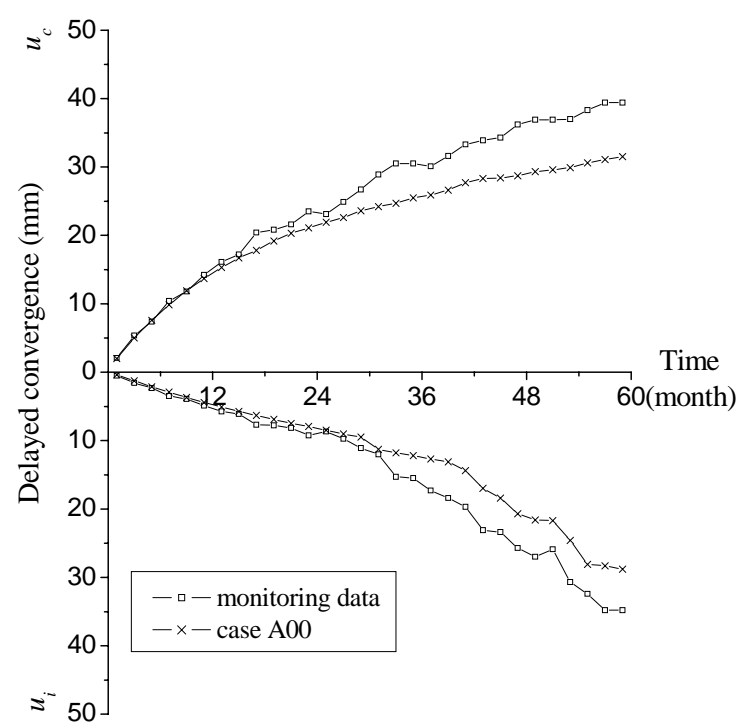

Fig. 8. Comparison of the monitoring data and the simulation results (case A00).

Similarly, the influence of viscoplastic parameters $G^{K}, \eta^{K}$ and $\eta^{M}$ is highlighted in case group B, where the strength deterioration parameters are assumed constant and the viscoplastic parameters are taken some random values. The weighted average parameters $\vec{b}^{00}$ can be obtained from the similar back-analyses process, and the simulation results of weighted average case B00 are delineated in Fig. 9. The case information of case group B are summarized in Table 4. It can be concluded that the viscoplastic parameters influence both the crown settlement and invert upheaval to some extend.

Table 4. Case information of group B.

\begin{tabular}{c|cccccc}
\hline $\begin{array}{l}\text { Case } \\
\text { No. }\end{array}$ & $\begin{array}{l}G^{K} \\
(\mathrm{MPa})\end{array}$ & $\begin{array}{l}\eta^{K} \\
(\mathrm{~Pa} \cdot \mathrm{s})\end{array}$ & $\begin{array}{l}\eta^{M} \\
(\mathrm{~Pa} \cdot \mathrm{s})\end{array}$ & $\begin{array}{l}e_{u c} \\
(\%)\end{array}$ & $\begin{array}{l}e_{u i} \\
(\%)\end{array}$ & $\begin{array}{l}\text { Weight } \\
(\%)\end{array}$ \\
\hline B01 & 800 & $3.0 \mathrm{e} 16$ & $8.0 \mathrm{e} 17$ & 3.9 & 2.8 & 16.6 \\
B02 & 700 & $4.0 \mathrm{e} 16$ & $7.0 \mathrm{e} 17$ & 1.5 & 9.2 & 10.3 \\
B03 & 600 & $5.0 \mathrm{e} 16$ & $6.0 \mathrm{e} 17$ & 0.9 & 13.5 & 7.7 \\
B04 & 500 & $6.0 \mathrm{e} 16$ & $5.0 \mathrm{e} 17$ & 1.2 & 7.9 & 12.1 \\
B05 & 400 & $7.0 \mathrm{e} 16$ & $4.0 \mathrm{e} 17$ & 2.0 & 11.1 & 8.4
\end{tabular}




\begin{tabular}{c|cccccc} 
B06 & 860 & $5.4 \mathrm{e} 16$ & $4.3 \mathrm{e} 17$ & 5.6 & 2.4 & 13.9 \\
B07 & 740 & $9.5 \mathrm{e} 16$ & $9.2 \mathrm{e} 17$ & 15.9 & 1.8 & 6.2 \\
B08 & 620 & $6.2 \mathrm{e} 16$ & $8.3 \mathrm{e} 17$ & 5.1 & 4.3 & 11.8 \\
B09 & 530 & $2.5 \mathrm{e} 16$ & $4.1 \mathrm{e} 17$ & 4.2 & 16.7 & 5.3 \\
B10 & 420 & $7.5 \mathrm{e} 16$ & $6.3 \mathrm{e} 17$ & 4.6 & 9.8 & 7.7 \\
\hline B00 & 644 & $5.4 \mathrm{e} 16$ & $6.3 \mathrm{e} 17$ & 1.0 & 8.9 & \\
\hline \multicolumn{6}{l}{ Group B: $\omega_{c}=0.05 \mathrm{MPa} / \mathrm{y}, \omega_{\phi}=3.0, R_{\text {thr }}=0.65$}
\end{tabular}

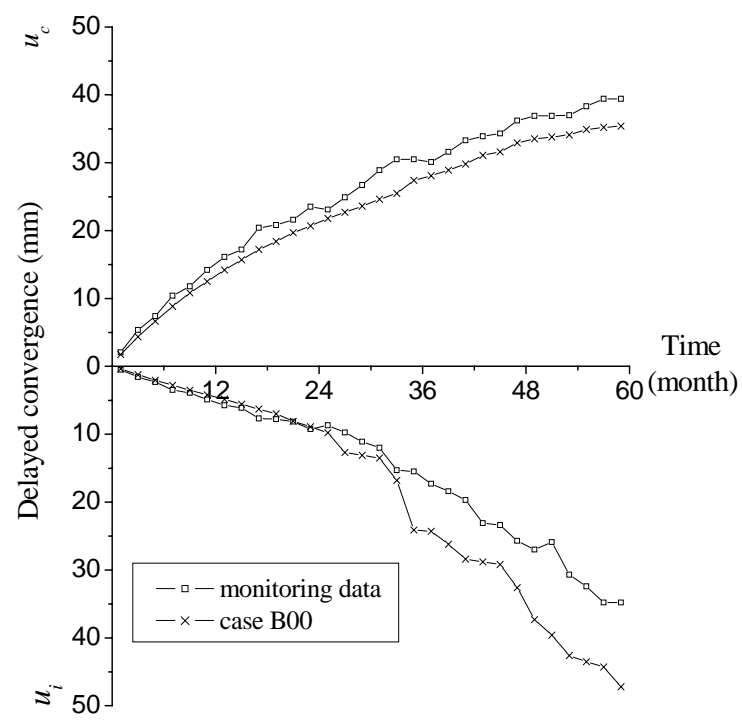

Fig. 9. Comparison of the monitoring data and the simulation results (case B00)

In case group $\mathrm{C}$, another 10 cases with different rheological parameters are studied, and the similar weighted average process is conducted to evaluate the "realistic" parameters $\vec{C}^{00}$ for the proposed model according to the in-sit monitoring data. Then, employing $\vec{c}^{00}$ again into the same simulations, the simulation results, as delineated in Fig. 10, agree with the monitoring data well and the errors fall below an acceptable range (see the case information summaries in Table 5). Therefore, the proposed model together with its corresponding parameters can account for the delayed deformation mechanics in Ureshino Tunnel Line I during its service time of first five years, and can be used to predict the further deformation of rock mass and help the maintenance of tunnel in the future.

Table 5. Case information of group C.

\begin{tabular}{cccccccccc}
\hline $\begin{array}{c}\text { Case } \\
\text { No. }\end{array}$ & $\begin{array}{c}G^{K} \\
(\mathrm{MPa})\end{array}$ & $\begin{array}{c}\eta^{K} \\
(\mathrm{~Pa} \cdot \mathrm{s})\end{array}$ & $\begin{array}{c}\eta^{M} \\
(\mathrm{~Pa} \cdot \mathrm{s})\end{array}$ & $\begin{array}{c}\omega_{c} \\
(\mathrm{MPa} / \mathrm{y})\end{array}$ & $\begin{array}{c}\omega_{\phi} \\
(\% / \mathrm{y})\end{array}$ & $\begin{array}{c}R_{\text {thr }} \\
e_{u c} \\
(\%)\end{array}$ & $\begin{array}{c}e_{u i} \\
(\%)\end{array}$ & $\begin{array}{c}\text { Weight } \\
(\%)\end{array}$ \\
\hline C01 & 800 & $3.0 \mathrm{e} 16$ & $8.0 \mathrm{e} 17$ & 0.103 & 5.46 & 0.45 & 3.0 & 72.5 & 1.2 \\
$\mathrm{C} 02$ & 700 & $4.0 \mathrm{e} 16$ & $7.0 \mathrm{e} 17$ & 0.081 & 4.25 & 0.55 & 2.5 & 7.9 & 8.6 \\
$\mathrm{C} 03$ & 600 & $5.0 \mathrm{e} 16$ & $6.0 \mathrm{e} 17$ & 0.067 & 3.06 & 0.60 & 0.1 & 3.7 & 3.6 \\
C04 & 500 & $6.0 \mathrm{e} 16$ & $5.0 \mathrm{e} 17$ & 0.045 & 2.12 & 0.65 & 1.8 & 15.5 & 5.1 \\
$\mathrm{C} 05$ & 400 & $7.0 \mathrm{e} 16$ & $4.0 \mathrm{e} 17$ & 0.021 & 1.05 & 0.80 & 2.4 & 12.0 & 6.2 \\
$\mathrm{C} 06$ & 860 & $5.4 \mathrm{e} 16$ & $4.3 \mathrm{e} 17$ & 0.016 & 5.52 & 0.55 & 8.1 & 2.6 & 8.3 \\
C07 & 740 & $9.5 \mathrm{e} 16$ & $9.2 \mathrm{e} 17$ & 0.046 & 3.88 & 0.60 & 3.8 & 1.9 & 15.7 \\
C08 & 620 & $6.2 \mathrm{e} 16$ & $8.3 \mathrm{e} 17$ & 0.039 & 3.21 & 0.75 & 2.4 & 30.5 & 2.7 \\
C09 & 530 & $2.5 \mathrm{e} 16$ & $4.1 \mathrm{e} 17$ & 0.059 & 0.95 & 0.85 & 1.2 & 2.1 & 27.2
\end{tabular}




\begin{tabular}{rrrrrrrrrr}
$\mathrm{C} 10$ & 420 & $7.5 \mathrm{e} 16$ & $6.3 \mathrm{e} 17$ & 0.096 & 1.37 & 0.70 & 69.9 & 5.8 & 1.2 \\
\hline $\mathrm{C} 00$ & 617 & $5.2 \mathrm{e} 16$ & $5.8 \mathrm{e} 17$ & 0.054 & 2.76 & 0.67 & 0.5 & 3.2 & \\
\hline
\end{tabular}

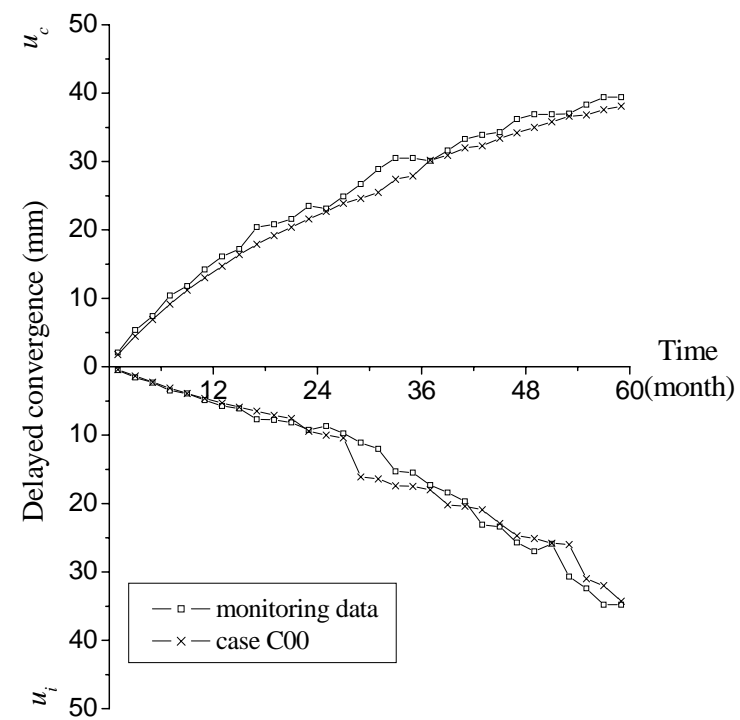

Fig. 10. Comparison of the monitoring data and the simulation results (case C00)

\section{Conclusions}

The time-dependent features of rock mass, which are called rheology generally, should be taken into account in the long-term design and maintenance of mountain tunnels. Based on the classic Burger-MC model, a Burger-Deterioration rheological model consisting of a Kelvin unit, a Maxwell unit and a deteriorating MC unit that are connected in series is proposed in this paper. A deterioration threshold and two deterioration ratios are introduced in this model to consider the time-dependent strength deterioration aspect of rock mass, without regarding that whether the loss of strength is caused by cycle loading fatigue, by clay mineral hydration or by other reasons.

The proposed model is implemented in the numerical codes FLAC ${ }^{3 \mathrm{D}}$ and applied to Ureshino Tunnel Line I, Nagasaki, Japan to account for the delayed deformations that occurred after its completion. The deformation characteristics of Kelvin unit, Maxwell unit and deteriorating MC unit are first studied respectively. The strain rates in these three units attenuate, stabilize and accelerate respectively with the rheological time, which correspond to the first, the second and the tertiary creep phases in some creep tests. When combining these three units in series, the deformation characteristics of the Burger-Deterioration model are conspicuously featured by its stair-typed increasing of convergence at the invert, which qualitatively agrees with the in-site monitoring data better than other rheological models. In addition, the parameters involved in the proposed model can be back-analyzed via a simplified weighted average method, according to the in-site monitoring data. The proposed model, together with the "realistic" parameters after back-analyses, can account for 
the delayed deformation mechanics after the tunnel was put into use, and can be applied to predict the further deformation of rock mass and help the maintenance of tunnel in the future.

\section{Appendix A}

Generally, the rheological parameters are not available for a certain engineering instance and only could take reference to literature on this occasion. Base on some test results on the rock specimens, the rheological parameters employed in this paper are evaluate in the following means. Two deterioration ratios can be evaluated by the Eq. (A1):

$$
\omega_{c}=\frac{c-c_{r e s}}{T_{c}}, \quad \omega_{\phi}=\frac{\phi-\phi_{\text {res }}}{T_{c}}
$$

In the above equations, cohesion, friction angle and their residual counterparts are available from the triaxial tests on Ureshino Tunnel rock specimens and listed in Table 1 (Japan Highway Society. 2000). $T_{c}$ is the expected creep lifetime and taken reference to laboratory tests (Shin et al., 2005). As for other rheological parameters, $\eta^{K}$ and $G^{K}$ can be obtained from conventional relaxation tests, $\eta^{K}$ from conventional creep tests and $R_{\text {thr }}$ from quasistatic compression tests ( $\mathrm{Li}$ and Xia, 2000; Shin et al., 2005).

Then using these rheological parameters from literature as "basic" properties (as listed in case A04, B04 and C04) and expanding them by random extrapolation, which generates the properties employed in the other nine cases (as listed in Table 3, Table 4 and Table 5). Then comparing the simulation results of each case to the monitoring data, using the weighted average means to calibrate the "basic" properties into the "realistic" properties as listed in case A00, B00 and C00.

\section{Reference}

Carranza-Torres, C. and Fairhurst, C. 1999. The elasto-plastic response of underground excavations in rock masses that satisfy the Hoek-Brown failure criterion. Int. J. Rock Mech. Min. Sci., 36 (6), 777-809.

Bhandari, A. and Inoue, J. 2005. Continuum damage mechanics for hysteresis and fatigue of quasi-brittle materials and structures. Int. J. Numer. Anal. Meth. Geomech., 29 (11), 1087-1107.

Fabre, G. and Pellet, F. 2006. Creep and time-dependent damage in argillaceous rocks. Int. J. Rock Mech. Min. Sci., 43 (6), 950-960.

Guan, Z., Jiang, Y. and Tanabashi, Y. 2006. Ground reaction analyses in conventional tunnelling excavation. Tunnelling and Underground Space Technology, 22 (2), 230-237

Haykin, S. 2002. Neutral Network - A Comprehensive Foundation. Prentice Hall, Inc.

Hudson, J. and Harrison, J. 1997. Engineering rock mechanics. London, Pergamon.

Itasca Consulting Group. 1997. FLAC3D, Fast Lagrange Analysis of Continua in 3 Dimensions, Version 2.0, User 
Manual. Minneapolis.

Japan Highway Society. 2000. The construction and monitoring reports of the Nagasaki Expressway (in Japanese).

Li, Y. and Xia, C. 2000. Time-dependent tests on intact rocks in uniaxial compression. Int. J. Rock Mech. Min. Sci., 37 (3), 467-475.

Maranini, E. and Brignoli, E. 1999. Creep behaviour of a week rock: experimental characterization. Int. J. Rock Mech. Min. Sci., 36 (1), 127-138.

Oreste, P. 2003. Analysis of structural interaction in tunnels using the convergence confinement approach. Tunnelling and Underground Space Technology, 18 (4), 347-363.

Pellet. F., Hajdu, A., Deleruyelle, F. and Besnus, F. 2005. A viscoplastic model including anisotropic damage for the time dependent behaviour of rock. Int. J. Numer. Anal. Meth. Geomech., 29 (9), 941-970.

Pichler, B. Lackner, R and Mang, H. 2003. Back analysis of model parameters in geotechnical engineering by means of soft computing. Int. J. Numer. Meth. Engng. 57 (14), 1943-1978.

Shao, J., Chau, K. and Feng, X. 2006. Modeling of anisotropic damage and creep deformation in brittle rocks. Int. J. Rock Mech. Min. Sci., 43 (4), 582-592.

Shin, K., Okubob, S., Fukuib, K. and Hashibab, K. 2005. Variation in strength and creep life of six Japanese rocks. Int. J. Rock Mech. Min. Sci., 42 (2), 251-260. 